\title{
Les Effets Indésirables Digestifs De La Chimiothérapie : Cas Des Patients De L'institut National D’oncologie De Rabat (Maroc)
}

\author{
Mustapha Ahtit \\ Laboratoire de Génétique et Biométrie, Faculté des Sciences, \\ Université Ibn Tofail, Kénitra, Maroc \\ Soulaymani abdelmajid \\ Khadmaoui Abderrazak \\ Laboratoire de Génétique et Biométrie, \\ Faculté des Sciences, Université Ibn Tofail, Kénitra, Maroc
}

\section{R. Benkirane}

Centre Anti Poison et de Pharmacovigilance du Maroc (CAPM), Rabat, Maroc

\section{Soulaymani Bencheikh Rachida}

Centre Anti Poison et de Pharmacovigilance du Maroc (CAPM), Rabat, Maroc

Faculté de Médecine et de Pharmacie, Université Mohammed V, Rabat, Maroc

\section{E. Kerak}

Équipe de virologie, cancérologie et Qualité Totale

Faculté des Sciences et Techniques, Mohammedia, Maroc

doi: 10.19044/esj.2016.v12n33p454 URL:http://dx.doi.org/10.19044/esj.2016.v12n33p454

\begin{abstract}
An adverse reaction of an anticancer drug is a harmful and an unintended reaction by patient suffering from cancer disease who is often polymedicated. The treatment of cancers by anti-cancer molecules produces serious adverse drug effects. The main purpose of this paper is to present and evaluate the digestive adverse effects involved in anti-cancer drugs and their potential of correlation to anticipate, prevent and improve the quality of care of patient suffering from cancer disease.

This is a prospective study that that enrolled 147 patients seen between January 25 and June 25, 2009 with adverse drug reactions due to an anticancer treatment. Breast and cavum cancers present 34\% of cases. The average age was 46.52 years. The sex ratio ( $\mathrm{M} / \mathrm{F}$ ) was 0.33 . During the study period 283 Adverse drug effects of anticancer drugs were collected with a predominance (132) adverse digestive effects and 32 anticancer drugs
\end{abstract}


were counted on all medical prescriptions. Nausea and vomiting are the most common side effects, sometimes very severe.

The availability of anti-emetics in the family of 5-HT3 serotoninergic antagonists has considerably improved the experience of patients undergoing anticancer chemotherapy, hence the importance of pharmacovigilance as a tool is to improve the quality of anticancer care.

Keywords: Digestive adverse effects, anticancer drugs, Chemotherapy, Cancers, supported.

\section{Résumé}

Un effet indésirable d'un médicament anticancéreux est une réaction nocive et non voulue par le patient atteint de cancer qui est, souvent, polymédiqué. Le traitement des cancers par les molécules anticancéreuses présente de nombreux effets indésirables médicamenteux. L'objectif de cet article est de présenter et d'évaluer les effets indésirables digestifs liés aux anticancéreux ainsi que leurs degrés de corrélation pour pouvoir les anticiper, les prévenir et améliorer, par conséquent, la qualité de prise en charge des patients cancéreux.

IL s’agit d'une étude prospective qui a inclus 147 patients rencontrés entre 25 janvier et 25 juin 2009 et présentant des effets indésirables médicamenteux suite à un traitement anticancéreux. Les cancers du sein et du cavum présentent 34\% des cas. L’âge moyen était de 46.52 ans. Le sexratio (M/F) était de 0 .33. Durant la période d'étude 283 EIM ont été colligés avec une prédominance (132) effets indésirables digestifs et on a compté 32 anticancéreux sur l'ensemble des ordonnances médicales. Les effets indésirables fréquents sont des nausées et vomissements, parfois très sévères. La mise à disposition des anti-émétiques de la famille des antagonistes sérotoninergiques 5-HT3 a considérablement amélioré le vécu des patients soumis à des chimiothérapies anticancéreuses, d'où l'importance de la pharmacovigilance comme outil d'amélioration de la qualité des soins anticancéreux.

Mots clés : Effets indésirables digestifs, anticancéreux, Chimiothérapie, Cancers, prise en charge.

\section{Introduction}

La sécurité d’emploi des médicaments anticancéreux représente un des grands problèmes contemporains de la santé publique, d’où la nécessite d'un suivi très rigoureux par la pharmacovigilance. De nombreuses études ont démontré que la toxicité liée à l'utilisation des médicaments en générale représentait un problème majeur dont les professionnels de santé et les 
patients étaient de plus en plus conscients. La saisie des annonces spontanées d'effets indésirables reste le meilleur instrument de détection de tels problèmes. Dans son rapport annuel portant sur l'année 2014, l’Agence nationale Française de sécurité du médicament témoigne de 46497 effets indésirables déclarés par les centres régionaux de pharmacovigilance (Marie Bonnet, octobre 2016).

Les effets indésirables médicamenteux liés aux molécules anticancéreuses utilisées dans les traitements des cancers sont nombreux et de divers types (Vidal ${ }^{\circledR}$, CNHIM, AFSSAPS). D’une façon générale, la fréquence de survenue de ces effets indésirables est variable en fonction du type de protocole de chimiothérapie. Il existe par ailleurs une grande variabilité entre les individus en ce qui concerne la tolérance d'une chimiothérapie donnée de telle sorte qu’il n’est pas possible de prédire de façon fiable sa tolérance pour un individu donné (Bruno Buecher, 2009).

Ces toxicités sont responsables d'une augmentation significative de la morbidité et de la mortalité. En effet, il est démontré que les effets indésirables médicamenteux sont responsables de près de $5 \%$ des hospitalisations (Howard RL et al, 2006) et de 0,15\% des décès (Pirmohamed $\mathrm{M}$ et al, 2004). Les EIM touchent 5 à 7\% de la population générale (Blenkinsop A et al, 2006) et 10 à 20\% des patients hospitalisés (Davies EC et al, 2006). Par ailleurs, le nombre de notifications des effets indésirables demeure en augmentation (estimée à 45\% entre 1998 et 2005 selon une étude anglaise (Patel $\mathrm{H}$ et al, 2007) et ceci est attribué non seulement au changement de la pratique médicale mais aussi au développement des systèmes de pharmacovigilance mis en place.

La fréquence des effets indésirables digestifs varient d'un patient à l'autre en fonction de différents facteurs de risque. Certains de ces facteurs sont liés au traitement antinéoplasique lui-même, d'autres dépendent plus directement du sujet. Ainsi, le risque d'apparition de vomissements se situe entre $60 \%$ et $100 \%$ des patients traités par les sels de platine (ex. : le cisplatine et le Carboplatine), entre $30 \%$ et $60 \%$ par le cyclophosphamide et chez $10 \%$ à $30 \%$ des patients traités par le 5FU (Vincent GIROD, Laurent GRELOT, Août 2001). Les chimiothérapies anticancéreuses induisent indirectement des crises émétiques qualifiées d'anticipées. Ces accès émétiques anticipés, présentés dès la seconde cure chimiothérapique, s'observent chez $25 \%$ à $60 \%$ des patients selon la nature du traitement prescrit (Andrykowski MA, 1990).

Très peu de données sur la tolérance des anticancéreux chez les patients traités aux différents services de l'institut national d'oncologie de Rabat sont disponibles, d'où l'importance de la surveillance des effets indésirables médicamenteux dans cet établissement de renommé. Nous nous sommes intéressés dans cette étude aux cytotoxiques utilisés en 
chimiothérapie anticancéreuse pour évaluer les différents effets indésirables digestifs observés. La connaissance des effets indésirables inhérents à ces médicaments est importante pour l'identification du médicament responsable et l'établissement d'une démarche pratique envers cette situation clinique.

\section{MÉTHODOLOGIE :}

Le présent travail consiste en une étude prospective menue auprès des oncologues et des patients cancéreux dans les services de consultation et de chimiothérapie à l'Institut National d'Oncologie de Rabat, le premier centre de référence, spécialisé dans le traitement des maladies cancéreuses au Maroc. La population concernée par cette étude est constituée :

- Des patients hospitalisés au service chimiothérapie de l'hôpital durant la période de l'étude et présentant un EIM ;

- Des patients ayant eu une prolongation de leur séjour hospitalier pour un effet indésirable durant la période de l'étude ;

- Des patients consultant au centre de consultation et présentant un EIM ;

- Des patients hospitalisés pour une pathologie dont la cause est susceptible être médicamenteuse.

Deux enquêteurs du Centre National de Pharmacovigilance (CMPV) ont visité tous les jours les services de l'hôpital concernés par cette étude pour collecter les événements indésirables et cela en coordination avec les professionnels de santé de l'INO.

Les documents utilisés pendant cette éludes sont :

- Fiche de recueil de pharmacovigilance : fiche standard de déclaration des effets indésirables des médicaments conçue par le CMPV ;

- Dossier Médical: Renseignements sur le patient sujet d’EIM démographiques, antécédents, histoire de la maladie, bilan para clinique, traitement reçu, évolution...

- Registre du service: Données administratives de tous les patients hospitalisés (sexe, âge, diagnostic, évolution, durée d'hospitalisation)

L’imputabilité des cas collectés est faite au niveau du CMPV selon la méthode française, Beni chou.

La saisie sur application Excel et l'analyse des résultats a été fait en coopération avec les responsables du Centre antipoison et de pharmacovigilance du Maroc et les professeurs du laboratoire de génétique et de biométrie à la faculté des sciences de l'université Ibn-Tofail, Kenitra, Maroc. L'application SPSS est l'outil utilisé pour analyser la base de données. 


\section{RÉSULTATS :}

III-1. Étude descriptive de la population étudiée :

\section{III-1. 1- caractéristiques épidémiologiques :}

Au cours de cette étude, qui s’est étalée sur une période de 6 mois, 147 patients présentant des effets indésirables suite à une ou plusieurs cures de chimiothérapie ont été recensés.

Tableau 1 : caractéristiques épidémiologiques de la population étudiée :

$\begin{array}{lll}\text { Variable étudié } & \text { Nombre de patient } & \text { \% } \\ \text { Tranches d’âge : } & & \\ \quad \begin{array}{l}\text { Inférieur à 35ans } \\ \text { Égale ou supérieur à 35ans inférieur à }\end{array} & 26 & 17,69 \\ \text { 60ans } & 104 & 70,75 \\ \quad \begin{array}{l}\text { Égale ou supérieur à 60ans } \\ \quad \text { Total }\end{array} & 17 & 11,56 \\ \text { Sexe } \quad & \mathbf{1 4 7} & \mathbf{1 0 0} \\ \quad \text { Féminin } & & \\ \quad \text { Masculin } & 81 & 55,1 \\ \quad \text { Inconnu } & 27 & 18,37 \\ \quad \text { Total } & 39 & 26,53 \\ \text { Type de cancer } & \mathbf{1 4 7} & \mathbf{1 0 0} \\ \quad \text { Sein } & & \\ \quad \text { Cavum } & 38 & 25,85 \\ \quad \text { Colorectal } & 16 & 10,88 \\ \text { Estomac } & 11 & 7,48 \\ \text { Poumon } & 9 & 6,12 \\ \quad \text { Maladie de hodgkin } & 9 & 6,12 \\ \text { Utérus } & 16 & 10,88 \\ \text { Os } & 5 & 3,4 \\ \text { Autres cancers } & 5 & 3,4 \\ \text { Total } & 38 & 25,85 \\ & \mathbf{1 4 7} & \mathbf{1 0 0}\end{array}$

L'âge de la population étudiée est entre 17ans et 77ans, 70,75\% d'entre eux se situe entre 35 et 60 ans avec un pic de 31(21,08\%) cas qui se trouve entre 50 ans et 55ans. La population étudiée a concerné 27 hommes, 81 femmes et 39 cas de sexe non renseignés sur les fiches d'enregistrement, le sexe ratio égal à 0,33 en faveur du sexe féminin. Les cancers du sein, du cavum, de maladie Hodgkin, colorectaux, du poumon, et de l'estomac représentent $67.33 \%$ des patients inclus dans l'étude. Le cancer du sein constitue à lui seul plus d'un quart de la population étudiée (25,85\%).

III-1. 2- les types de la toxicité observée :

Le nombre et la nature des effets indésirables médicamenteux recensés au cours de cette étude sont présentés dans le tableau 2. 
Tableau 2 : Types de toxicité observés chez les patients inclus dans l'étude

$\begin{array}{lll}\text { Type de toxicité } & \text { Nombre } & \mathbf{( \% )} \\ \text { Toxicité digestive } & 132 & 55.46 \\ \text { Toxicité peau, phanères } & 61 & 25.63 \\ \text { Toxicité médullaire } & 25 & 10.50 \\ \text { Toxicité rénale } & 8 & 3.36 \\ \text { Toxicité pulmonaire } & 6 & 2.52 \\ \text { Toxicité métabolique } & 4 & 1.68 \\ \text { Toxicité sur les gonades } & 2 & 0.84 \\ \text { TOTAL } & \mathbf{2 3 8} & \mathbf{1 0 0}\end{array}$

Ainsi, on a pu recenser 238 effets indésirables de différentes natures suite au traitement des patients par la chimiothérapie. Le tableau des résultats montre une prédominance de la toxicité digestive avec 55,46\%, suivis des toxicités sur la peau et les phanères avec $25.63 \%$ et en troisième position se trouvent les toxicités médullaires (10,50\%). Dans cette étude, on a pu dénombrer un total de 132 effets indésirables médicamenteux de type digestif notamment, et par ordre de fréquence, 83 cas de vomissements, 17 cas de diarrhées, 16 cas de mucites, 10 cas de nausées et 6 cas de constipations.

\section{III-2. -étude analytique de la liaison entre les médicaments et les effets indésirables digestifs :}

Nous avons opté pour une étude analytique via l'application de logiciel SPSS pour mettre en évidence l'importance de la liaison entre les médicaments prises comme traitement de cancer et les effets indésirables digestifs recensés. A cet effet, nous avons croisé chaque médicament avec chaque effet indésirable pour calculer le Qhi-Deux (X2), le risque relatif(RR), l'intervalle de confiance à 95\% (IC à 95\%) et le degré de signification (P). Un grand nombre de tableaux de croisement ont été mis en évidence, et comme notre article s'intéresse aux effets indésirables digestifs, nous avons éliminé tous les résultats concernant les autres types d'effets, ainsi que ceux des effets digestifs dont la liaison n'est pas hautement significative ou très significative. Le tableau 3 , représente les médicaments influençant l'apparition des effets indésirables digestifs chez les patients intoxiqués.

Tableau 3 : La corrélation entre les effets indésirables digestifs et les médicaments anticancéreux. :

$\begin{array}{llllll}\text { Médicament } & \text { EIM } & \text { X2 } & \text { P } & \text { RR } & \text { IC } \\ \text { Doxorubicine } & \text { Vomissement } & 17.27 & 0.000 & 5.938 & 2.419-14.571 \\ \text { Gemcitabin } & \text { Constipation } & 17.057 & 0.000 & 23.000 & 2.970-178.092 \\ \text { Méthotrexate } & \text { Diarrhées } & 15.505 & 0.000 & 0.103 & 0.064-0.167 \\ \text { Rituximab } & \text { Constipation } & 10.920 & 0.001 & 28.000 & 1.523-514.922 \\ \text { Epirubicine } & \text { Mucites } & 9.825 & 0.002 & 18.571 & 1.582-218.018 \\ \text { Cyclophosphamide } & \text { Mucites } & 4.841 & 0.028 & 3.330 & 1.088-10.191 \\ \text { Gemcitabin } & \text { Nausées } & 8.997 & 0.003 & 11.167 & 1.627-76.637\end{array}$


$\mathrm{X}^{2}$ : khi-deux ; RR : Risque relatif ; IC95\% : Intervalle de confiance à 95\% et P : degré de signification ( $\mathrm{P}>0,05$; liaison non significative ; $0,01<\mathrm{p} \leq 0,05$ : liaison significative (5\%) ; $0,001<p \leq 0,01$ : liaison très significative $(1 \%) ; p \leq 0,001$ : liaison hautement significative).

$\mathrm{Au}$ total, 7 médicaments sont incriminés de façon hautement significative et très significative dans l'apparition des effets indésirables digestifs. Ainsi, la liaison est hautement significative pour vomissement doxorubicine, constipation - gemcitabin, diarrhées - méthotrexate et constipation-rituximab. Elle est très significative pour mucites-epirubicine, mucites cyclophosphamide et nausées-gemcitabin.

\section{III-3. -Les effets indésirables digestifs : fréquence et médicament incriminé dans son apparition.}

III-3. 1-Vomissement :

Les vomissements constituent une manifestation d'expression digestive fréquente isolée ou souvent associée à d'autres symptômes d'emblée évidents ou à rechercher. Il faut distinguer les formes aiguës et chroniques. Les vomissements aigus peuvent être d'origine digestive et accompagnés notamment une pathologie abdominale aiguë ; ils peuvent être la manifestation d'une pathologie viscérale aiguë (vomissements reflexes). Ils peuvent être secondaires à des stimuli chimiques, vestibulaires, ou cérébraux. Les vomissements aigus sont donc très peu spécifiques. Il convient d'intégrer ce symptôme en fonction des divers stimuli capable de stimuler le centre des vomissements (Fournet, 2003).

L'étude a mis en évidence que 83 patients sur 147 ont présenté les vomissements avec une fréquence de $56,50 \%$ (voir tableau 4) et que le médicament doxorubicine est hautement lié à l'apparition de cette toxicité, avec un degré de signification de 0,000 (haute signification) (voir tableau 5).

Tableau 4 : fréquence des vomissements au sein de la population étudiée :

Vomissement

$\begin{array}{lllll} & \text { Effectifs } & \text { \% } & \text { \% valide } & \text { \% cumulé } \\ \text { Absence } & 64 & 43,5 & 43,5 & 43,5 \\ \text { Présence } & 83 & 56,5 & 56,5 & 100,0 \\ \text { Total } & 147 & 100,0 & 100,0 & \end{array}$

Tableau 5 : La corrélation des vomissements avec les médicaments anticancéreux :

$\begin{array}{llllll}\text { Médicament } & \text { EIM } & \text { X2 } & \text { P } & \text { RR } & \text { IC } \\ \text { Doxorubicine } & \text { Vomissement } & 17.27 & 0.000 & 5.938 & 2.419-14.571\end{array}$

\section{III-3. 2-Nausées :}

La nausée est un malaise dans l'estomac ou un spasme qui commence dans l'estomac et se prolonge jusqu'à la gorge. Les nausées peuvent également provoquer une transpiration, des étourdissements ou une faiblesse générale, ainsi qu'une salivation supérieure à la normale. Elles peuvent 
survenir même lorsque la personne affectée ne pense pas à des aliments et entraîner des haut-le-cœur et des vomissements (American Cancer Society, 2015).

L’étude a montré que 10 patients sur 147 ont présenté les nausées avec une fréquence de 6,8\% (voir tableau 6) et que le médicament gemcitabin est très lié à l'apparition de cette toxicité, avec un degré de signification de 0,003 (tés significative) (voir tableau 7).

Tableau 6 : fréquence des nausées au sein de la population étudiée :

Nausées

$\begin{array}{lllll} & \text { Effectifs } & \% & \% \text { valide } & \% \text { cumulé } \\ \text { Absence } & 137 & 93,2 & 93,2 & 93,2 \\ \text { Présence } & 10 & 6,8 & 6,8 & 100,0 \\ \text { Total } & 147 & 100,0 & 100,0 & \end{array}$

Tableau 7 : La corrélation des nausées avec les médicaments anticancéreux :

$\begin{array}{lllllll}\text { Médicament } & & \text { EIM } & \text { X2 } & \text { P } & \text { RR } & \text { IC } \\ \text { Gemcitabin } & & \text { Nausées } & 8.997 & 0.003 & 11.167 & 1.627-76.637\end{array}$

\section{III-3. 3-Diarrhées :}

La diarrhée se caractérise par des selles liquides qui sont plus fréquentes qu'à l'habitude (3 fois ou plus par jour). Elle peut être accompagnée de doleurs dans l'abdomen, de ballonnement et de crampes au ventre (Centre hospitalier de l'université de Montréal, mai 2014).

Le tableau ci-dessous montre que : 17 patients sur 147 ont présenté des diarrhées avec une fréquence de 11,6\% (voir tableau 8) et le médicament méthotrexate est hautement lié à l'apparition de cette toxicité, avec un degré de signification de 0,000 (hautement significative) (voir tableau 9).

Diarrhées

Tableau 8 : fréquence des diarrhées au sein de la population étudiée :

$\begin{array}{lllll} & \text { Effectifs } & \text { \% } & \text { \% valide } & \text { \% cumulé } \\ \text { Absence } & 130 & 88,4 & 88,4 & 88,4 \\ \text { Présence } & 17 & 11,6 & 11,6 & 100,0 \\ \text { Total } & 147 & 100,0 & 100,0 & \end{array}$

Tableau 9: La corrélation des diarrhées avec les médicaments anticancéreux

\begin{tabular}{|c|c|c|c|c|c|}
\hline Médicam & EIM & $\mathrm{X} 2$ & $\mathbf{P}$ & RR & IC \\
\hline Méthotrexate & Diarrhées & 15.505 & 0.000 & 0.103 & 0.064-0.167 \\
\hline
\end{tabular}

\section{III-3. 4- Mucites}

La mucite est une inflammation de la muqueuse qui recouvre l'intérieur des viscères et des cavités, en particulier la bouche. C'est une complication fréquente des traitements chimiothérapiques et radiothérapiques. La sévérité des symptômes dépend des traitements prescrits et de la sensibilité du patient, pouvant avoir des conséquences importantes sur la prise alimentaire. La mucite buccale est aussi appelée stomatite (Jean-Claude KARP) 
Cette étude a pu mettre en évidence que 10,9\% présentent de la mucite (voir tableau 10) et les deux médicaments à l'origine de cette toxicité sont, notamment, epirubicine et cyclophosphamide avec successivement des degrés de signification de 0,002 et de 0,028 (voir tableau 11).

Tableau 10 : fréquence des mucites au sein de la population étudiée : Mucites

$\begin{array}{lllll} & \text { Effectifs } & \text { \% } & \text { \% valide } & \text { \% cumulé } \\ \text { Absence } & 131 & 89,1 & 89,1 & 89,1 \\ \text { Présence } & 16 & 10,9 & 10,9 & 100,0 \\ \text { Total } & 147 & 100,0 & 100,0 & \end{array}$

Tableau 11 : La corrélation des mucites avec les médicaments anticancéreux

$\begin{array}{llllll}\text { Médicament } & \text { EIM } & \text { X2 } & \text { P } & \text { RR } & \text { IC } \\ \text { Epirubicine } & \text { Mucites } & 9.825 & 0.002 & 18.571 & 1.582-218.018 \\ \text { Cyclophosphamide } & \text { Mucites } & 4.841 & 0.028 & 3.330 & 1.088-10.191\end{array}$

III-3.5- Constipation :

La constipation est un symptôme qui correspond à une insatisfaction du malade lors de la défécation, due :

- soit à des selles peu fréquentes (moins de 3 selles hebdomadaires) ;

- soit à une difficulté pour exonérer ;

- soit aux deux.

Les difficultés pour exonérer peuvent se traduire par des efforts de poussée, une sensation de gêne au passage des selles ou d'évacuation incomplète, l'émission de selles dures, un temps d'exonération anormalement prolongé ou la nécessité de manœuvres digitales pour obtenir une vacuité rectale (La Collégiale des universitaires en hépato-gastro-entérologie, octobre 2012).

Cette étude a montré que 4,10\% des patients ayant présenté des cas de constipation (tableau 12) et les deux médicaments qui sont à l'origine de cette toxicité sont, notamment, gemcitabin et rituximab avec successivement des degrés de signification de 0,000 et de 0,001 (haute signification, tableau 13).

Tableau 12 : fréquence de la constipation au sein de la population étudiée :

Constipation :

$\begin{array}{lllll} & \text { Effectifs } & \text { \% } & \text { \% valide } & \text { \% cumulé } \\ \text { Absence } & 141 & 95,9 & 95,9 & 95,9 \\ \text { Présence } & 6 & 4,1 & 4,1 & 100,0 \\ \text { Total } & 147 & 100,0 & 100,0 & \end{array}$

Tableau 13 : La corrélation de la constipation avec les médicaments anticancéreux :

$\begin{array}{llllll}\text { Médicament } & \text { EIM } & \text { X2 } & \text { P } & \text { RR } & \text { IC } \\ \text { Gemcitabin } & \text { Constipation } & 17.057 & 0.000 & 23.000 & 2.970-178.092 \\ \text { Rituximab } & \text { Constipation } & 10.920 & 0.001 & 28.000 & 1.523-514.922\end{array}$




\section{DISCUSSION :}

Malgré la multiplicité et la précision des analyses auxquelles est soumis un médicament avant son enregistrement, certains effets indésirables parfois très graves n'apparaissent que lorsque le produit est commercialisé à grande échelle et consommé quotidiennement (Anna-Christina Zysset, 2004).

L’objectif de cet article est de présenter et d’évaluer les effets indésirables digestifs liés aux anticancéreux ainsi que leurs degrés de corrélation pour pouvoir les anticiper, les prévenir et améliorer, par conséquent, la qualité de prise en charge des patients cancéreux. 238 effets indésirables de différentes natures ont été recensé suite au traitement des patients cancéreux par la chimiothérapie. Le tableau des résultats montre une prédominance de la toxicité digestive avec 55,46\%, suivis des toxicités sur la peau et les phanères avec $25.63 \%$ et en troisième position se trouvent les toxicités médullaires $(10,50 \%)$.

La corrélation statistique entre les médicaments et les effets indésirables a montré qu'au moins 7 médicaments sont incriminés de façon hautement significative et très significative dans l'apparition des effets indésirables digestifs. Le tableau suivant synthétise les différentes liaisons qu'on a pu mettre en évidence dans notre étude :

$\begin{array}{cccc}\text { EIM constaté } & \text { Médicament responsable } & \mathbf{P} & \mathbf{\%} \\ \text { Vomissement } & \text { Doxorubicine } & 0,000 & 56,5 \\ \text { Nausées } & \text { Gemcitabin } & 0,000 & 6,8 \\ \text { Diarrhées } & \text { Méthotrexate } & 0,000 & 11,6 \\ \text { Mucites } & \text { Epirubicine } & 0.002 & 10,9 \\ & \text { Cyclophosphamide } & 0.028 & \\ \text { Constipation } & \text { Gemcitabin } & 0,000 & 4,1 \\ & \text { Rituximab } & 0,001 & \end{array}$

Ainsi, la liaison est hautement significative pour vomissement doxorubicine, constipation - gemcitabin, diarrhées - méthotrexate et constipation-rituximab. Elle est très significative pour mucites-epirubicine, mucites cyclophosphamide et nausées-gemcitabin.

Les données du résultat de notre étude [tableaux 1, 3] montre une importance significative des vomissements et des nausées qui atteint une fréquence de 63,3\%. Les données de la littérature montrent que les effets indésirables digestifs constituent la catégorie la plus fréquente suite à un traitement de chimiothérapie, ainsi, les effets indésirables aigus fréquents des cytotoxiques sont des nausées et vomissements, parfois très sévères. Ils s’observent chez 25 à $60 \%$ des patients selon la nature du traitement anticancéreux (Andrykowski MA, 1990). On retiendra également que l'administration à fortes doses d'agents cytotoxiques majore le potentiel émétique de la 
chimiothérapie, de même que l'association de différents agents (effet cumulatif) (Vincent GIROD, Laurent GRELOT, Août 2001).

Nous avons mis en évidence une liaison hautement significative entre le vomissement et le doxorubicine et très significative entre les nausées et la gemcitabin [tableaux 2, 7], chose plus ou moins confirmée par la littérature, ainsi, le risque d'apparition des nausées et vomissements est entre 31 à 90\% pour doxorubicine et entre 10 à $30 \%$ pour gemcitabin [M. Espié 2013].

Aussi, nous avons constaté des diarrhées dans 11 ,6\% des cas [tableau 8], avec une liaison hautement significative à la méthotrexate [tableau 9], $\mathrm{M}$. Espié, du Centre des maladies du sein, oncologie médicale, St Louis, dans sa présentation sur la surveillance des effets secondaires des traitements anticancéreux, consulté sur le site http://docplayer.fr/ le 25 Avril 2016, confirme plus au moins cette donnée de notre étude, ainsi, les diarrhées sont entre 5 à 15\% des traitements anti-cancéreux (par stimulation du péristaltisme : cisplatine, dacarbazine) ou par atteinte muqueuse (fluorouracile, capecitabine, méthotrexate, cytarabine) colite à clostridium associée au docetaxel.

Les constipations sont plus rares avec une fréquence de 4,10\% trouvée dans notre étude [tableau 9], et nous avons mis en évidence une liaison hautement significative avec la gemcitabin et la rituximab [tableau 13]. Les données de la littérature montrent l'existence d'une liaison entre la constipation et vincristine, vinorelbine, vindésine, vinblastine et à un moindre degré des taxanes (M. Espié 2013).

Parmi les soins oncologiques de support, la prise en charge des nausées et vomissements chimio-induits a connu des évolutions importantes ces deux dernières décennies. Une meilleure connaissance de la physiopathologie des vomissements et l'arrivée de deux classes d'antiémétiques à fort index thérapeutique, les antagonistes des récepteurs de type 3 à la sérotonine (anti-5HT3 ou sétrons) dans les années 1990 et les antagonistes des récepteurs de type 1 aux neurokinines (anti-NK1) en 2000, associés ou non aux corticoïdes, ont contribué à l'amélioration du contrôle de cet effet secondaire invalidant, toujours redouté des patients (J-P Durand, octobre 2009).

Le protocole d'administration de l'agent antinéoplasique doit aussi être pris en compte afin de limiter la sévérité des nausées et vomissements. Ainsi, l'administration par perfusion intraveineuse prolongée est moins émétique qu'une administration en perfusion courte ou en bolus. De même, les traitements chimiothérapiques à base de sels de platine semblent moins émétiques lorsqu'ils sont administrés en fin d'après-midi 


\section{Conclusion :}

L'emploi des molécules anticancéreuses demande un suivi très rigoureux et la saisie des annonces spontanées d'effets indésirables reste le meilleur instrument de détection de tels problèmes, d'où l'importance de la pharmacovigilance comme outil d'amélioration de la qualité des soins anticancéreux.

L’objectif de notre étude est de présenter et d'évaluer les effets indésirables digestifs liés aux anticancéreux ainsi que leurs degrés de corrélation afin de les anticiper, les prévenir et améliorer, par conséquent, la qualité de prise en charge des patients cancéreux.

Les effets indésirables digestifs constituent la catégorie la plus fréquente suite à un traitement de chimiothérapie avec une fréquence 55,46\%. Notre étude a permis de faire le lien entre les EIM digestifs et les médicaments responsables. Par conséquent, certains effets indésirables peuvent être limités ou évités grâce à des traitements préventifs, surtout avec la mise à disposition des anti-émétiques de la famille des antagonistes sérotoninergiques 5-HT3 qui a considérablement amélioré le vécu des patients soumis à des chimiothérapies anticancéreuses. Au-delà des solutions médicamenteuses antiémétiques, des conseils pratiques donnés avant le début du traitement, notamment, hygiéno-diététiques, permettent de limiter les nausées et les vomissements chimio-induits.

Le management de la qualité de prise en charge des cancéreux exige l'implantation de la Pharmacovigilance dans les centres d'oncologie et oblige la notification des effets indésirables liés aux médicaments par le professionnel de santé ou l'industriel qui en a connaissance, afin de promouvoir la sécurité d’emploi des médicaments anticancéreux et améliorer la confiance du patient dans les molécules autorisées sur le marché, et par conséquent améliorer la qualité des soins anticancéreux offerte aux usagers.

\section{References:}

1. Alexopoulou A. Adverse drug reactions as a cause of hospital admissions: A 6-month experience in a single center in Greece. Eur J Intern Med 2008.

2. American Cancer Society "Nausea and Vomiting”, consulté sur le site http://www.cancer.org, le 17/12/2015.

3. Andrykowski MA. The role of anxiety in the development of anticipatory nausea and vomiting: a review and synthesis. Psychosomatic Med 1990.

4. Anna-Christina Zysset, Sécurité des médicaments en Suisse, la Revue Mens Suisse Odondotonstomatol numéro d’octobre 2004.

5. Arrêté du 6 avril 2011 relatif au management de la qualité de la prise en charge médicamenteuse et aux médicaments dans les 
établissements de santé en France.

6. Blenkinsopp A, Wilkie P, Wang M, Routledge PA. Patient reporting of suspected adverse drug reactions: a review of published literature and international experience. Br J Clin Pharmacol 2006.

7. Bousquet PJ, Demoly $P$. Une synthèse sur l’épidémiologie des hypersensibilités médicamenteuses. Rev Fr Allergol Immunol 2005.

8. Bruno Buecher, Généralités sur les effets secondaires indésirables de la chimiothérapie consultée sur le site web de la Fédération Francophone de Cancérologie Digestive http://www.ffcd.fr, Janvier 2009.

9. Caroline battu, L'accompagnement nutritionnel d'un adulte présentant des nausées et des vomissements chimio-induits, science direct, volume 53, octobre 2014.

10. Centre hospitalier de l'université de Montréal, La diarrhée liée au traitement contre le cancer, société canadienne des cancers, consulté sur le site : chumontreal.qc.ca, en mai 2014.

11. Davies EC, Green CF, Mottram DR, Pirmohamed M. Adverse drug reactions in hospital in-patients, 2006.

12. Fiszenson-Albala F, Auzerie V, Mahe E et al. A 6-month prospective survey of cutaneous drug reactions in a hospital setting. 2003.

13. FOURNET Jacques Corpus Médical - Faculté de Médecine de Grenoble 1/7 les vomissements -Avril 2003. Éditions CEDH, 2003.

14. Howard RL, Avery AJ, Slavenburg S et al. Which drugs cause preventable admissions to hospital? A systematic review. Br J Clin Pharmacol 2006.

15. Hernandez-Salazar A, Rosales SP, Rangel-Frausto S, Criollo E, Archer-Dubon C, rozco-Topete R. Epidemiology of Adverse Cutaneous Drug Reactions. A Prospective Study in Hospitalized Patients. Arch Med Research 2006.

16. Howard RL, Avery AJ, Slavenburg S et al. Which drugs cause preventable admissions to hospital? A systematic review. Br J Clin Pharmacol 2006.

17. Jean-Claude KARP, Chimio ou radio-induites, Prise en charge par des médicaments homéopathiques, consultation d'homéopathie au Centre Hospitalier de Troyes, service d’Oncologie-Radiothérapie, consulté sur le site http://www.cedh.org/ le 26 /06/2016.

18. Jose J, Rao PGM. Pattern of adverse drug reactions notified by spontaneous reporting in an Indian tertiary care teaching hospital. Pharmacol Research 2006.

19. J-P Durand, I Madelaine, F Scotté, Recommandations pour la prévention et le traitement des nausées et vomissements 
induits par la chimiothérapie, Bulletin du cancer volume 96 numéro 10, Octobre 2009.

20. La Collégiale des universitaires en hépato-gastro-entérologie (CDUHGE), abrégé d’hépato-gastro-entérologie - 2ème édition - Partie « Connaissances » - Editions Elsevier-Masson -Octobre 2012.

21. M. Espié, Surveillance des effets secondaires des traitements anticancéreux, Centre des maladies du sein, oncologie médicale, St Louis, 2013.

22. Marie Bonnet, Pour une diffusion de la culture de l'effet indésirable, revue gestion hospitalière dans son numéro 559 d'octobre 2016.

23. Ministère de la santé au Maroc, Organisation du Système National de Pharmacovigilance, circulaire $\mathrm{n}^{\circ} 003$ du 04 janvier 2016.

24. Patel H, Bell D, Molokhia M, Srishanmuganathan J, Patel M, Car J, Majeed A. Trends in hospital admissions for adverse drug reactions in England: analysis of national hospital episode statistics 1998-2005. BMC Clin Pharmacol 2007.

25. Pirmohamed M, James S, Meakin S, Green C, Scott AK, Walley TJ, Farrar K, Park BK, Breckenridge AM. Adverse drug reactions as cause of admission to hospital: prospective analysis of 18820 patients. BMJ 2004.

26. Vincent GIROD, Laurent GRELOT, Physiopathologie des vomissements en chimiothérapie oncologique, In Medicine thérapeutique, Août 2001. 